\title{
Moving from vaginal hysterectomy to "no-incision" hysterectomy: how terminology has an impact
}

\author{
Michael Moen ${ }^{1}$ \\ Received: 30 October 2016 / Accepted: 30 October 2016 / Published online: 22 November 2016 \\ (C) The International Urogynecological Association 2016
}

It is estimated that one in three women undergo hysterectomy in the USA, with the majority of these procedures performed via open abdominal surgery [1]. It is well known that morbidity related to hysterectomy can be reduced by using less invasive surgical techniques, such as vaginal and laparoscopic approaches. In some countries, the proportion of vaginal hysterectomies is over $40 \%$ and in others, the proportion of laparoscopic hysterectomies is over $80 \%$, with a resultant decreased number of abdominal hysterectomies being performed [2, 3]. However, despite the availability of vaginal hysterectomy for over a century and that of laparoscopic hysterectomy for almost three decades, the most common procedure currently performed worldwide is still abdominal hysterectomy. Recently, robotic hysterectomy has been introduced and has been rapidly adopted in some countries, including the USA. Despite a lack of scientific evidence supporting its routine use, it is estimated that robotic hysterectomy now accounts for over $40 \%$ of hysterectomies in some areas of the USA [4].

The fact that there has been a concomitant drop in the abdominal hysterectomy rate would seem to vindicate the use of the robotic approach for hysterectomy, but use of the robot has also reduced the use of other minimally invasive surgery (MIS) techniques such as vaginal and laparoscopic hysterectomy [5]. With scientific evidence supporting the use of vaginal and laparoscopic hysterectomy, how is it that robotic hysterectomy has become the predominant approach? It cannot be denied that the aggressive industry-supported

Michael Moen

michael.moen@advocatehealth.com

1 Chicago Medical School/Rosalind Franklin University, Illinois Urogynecology, Ltd., 1875 Dempster Street, Suite 665, Park Ridge, IL 60068, USA marketing of robotic hysterectomy has influenced decisionmaking by patients, hospitals, surgeons. In fact, this has also resulted in the renewed marketing of traditional laparoscopic hysterectomy, which has had to coin the new term "straightstick laparoscopy" to differentiate it from "robotic-assisted laparoscopy".

Aggressive competition has now led to a myriad of new terms and acronyms (some of them actually trademarked), which leave patients, and even many surgeons, unclear on the specifics of each technique. In addition to the basic terms abdominal hysterectomy ( $\mathrm{AH})$, laparoscopic hysterectomy (LH), robotic hysterectomy $(\mathrm{RH})$, and vaginal hysterectomy (VH), the following terms have also appeared in recent medical literature and patient materials: da Vinci hysterectomy $(\mathrm{dH})$, da Vinci single-site hysterectomy (dSSH), laparoendoscopic single-site hysterectomy (LESS), robotic-assisted laparoscopic hysterectomy (RALH), robotic total vaginal NOTES [natural orifice translumenal endoscopic surgery] hysterectomy (RTVNH), robotic vaginally assisted NOTES hysterectomy (RVANH), single-incision laparoscopic surgery hysterectomy (SILS), single-site hysterectomy (SSH), total intrafascial laparoscopic hysterectomy (TAIL), vaginal access minimally invasive surgery hysterectomy (VAMIS), and vaginally assisted NOTES hysterectomy (VANH). This terminology seems to feed into our human nature that drives us to assume that newer, technological-sounding terms indicate something better.

In addition to the confusion created by the increased terminology and acronyms, there is also confusion created by the discrepancies and misinformation contained in patientdirected marketing materials. The most intense competitive marketing appears to be between straight-stick laparoscopy and robotic-assisted laparoscopy and is evident in patient education materials produced by the industry related to these two approaches. These marketing materials are often incomplete, 
misleading, and biased with an underlying intent to direct patients (and surgeons) to favor one approach over the other, despite scientific evidence that might suggest the better approach for an individual patient. Vaginal hysterectomy is mentioned in these materials, but is not accurately compared with the other MIS techniques. The fact that vaginal hysterectomy is actually the least invasive approach appears to get lost in the argument between laparoscopy and robotics.

This situation has caused me to wonder how vaginal hysterectomy would be marketed today if it had just been introduced as a new approach to hysterectomy. It likely wouldn't be referred to as "vaginal hysterectomy," as this involves two words that are not necessarily the most comfortable words to promote in a public forum. The goal is to reduce open abdominal hysterectomy by using minimally invasive techniques, and vaginal hysterectomy meets the criteria of a minimally invasive technique. And if we follow current marketing trends in hysterectomy focused on fewer and smaller abdominal incisions, an obvious point of fact is that the vaginal approach involves no abdominal incision. Therefore, in comparison with the other MIS approaches such as "single-site/single incision," it would be accurate to refer to vaginal hysterectomy as the "no-incision" hysterectomy. Using this terminology might result in a new look for vaginal hysterectomy in today's competitive hysterectomy marketing.

In our practice, it seems that at least once a week a patient who is an excellent candidate for vaginal hysterectomy listens to our recommendation and then asks "will you be using the robot to do my hysterectomy?" In the past, when we have explained that vaginal hysterectomy is actually less invasive than robotic hysterectomy, many patients have looked at us as if we must not know much about hysterectomies as we don't even seem to be aware of the benefits of the latest, greatest robotic hysterectomy technique that is sweeping the country. So now we have taken a new approach to hysterectomy terminology. Today, we tell patients that they are candidates for what is called a "no-incision hysterectomy," which is performed through a small incision hidden inside the vagina. This type of hysterectomy is actually the least invasive type of hysterectomy, even less invasive than belly-button surgery. In fact, it is so minimally invasive we don't even need the robot. For some reason, using this terminology seems to satisfy our patients that they are indeed getting a contemporary approach that is the best procedure for their individual situation. Vaginal hysterectomy is supported by scientific evidence, but it is not new and not well-marketed. As our patients seem to be more interested in new things, we can at least use an appropriate new term for this procedure. Hopefully by offering a "no-incision hysterectomy," our patients will see this option from the proper perspective to understand its value in comparison with the other hysterectomy techniques currently being suggested.

\section{References}

1. Wright JD, Herzog TJ, Tsui J, Ananth CV, Lewin SN, Lu YS, et al. Nationwide trends in the performance of inpatient hysterectomy in the United States. Obstet Gynecol. 2013;122:233-41.

2. Brummer THI, Jalkanen J, Fraser J, Heikkinen AM, Kauko M, Mäkinen J, et al. FINHYST 2006-national prospective 1-year survey of 5,279 hysterectomies. Hum Reprod. 2009;24:2515-22.

3. Donnez O, Jadoul P, Squifflet J, Donnez J. A series of 3190 laparoscopic hysterectomies for benign disease from 1990 to 2006: evaluation of complications compared with vaginal and abdominal procedures. BJOG. 2009;116:492-500.

4. Swenson CW, Kamdar NS, Harris JA, Uppal S, Campbell DA, Morgan DM. Comparison of robotic and other minimally invasive routes of hysterectomy for benign indications. Am J Obstet Gynecol. 2016;215:650.e1-8.

5. Smorgick N, Patzkowsky KE, Hoffman MR, Advincula AP, Song $\mathrm{AH}$, As-Sanie S. The increasing use of robot-assisted approach for hysterectomy results in decreasing rates of abdominal hysterectomy and traditional laparoscopic hysterectomy. Arch Gynecol Obstet. 2014;289:101-5. 